\title{
More Surprises in the General Theory of Lattice Systems
}

\author{
Alan D. Sokal \\ Courant Institute of Mathematical Sciences, New York University, 251 Mercer Street, New York, \\ NY 10012, USA
}

\begin{abstract}
I use Israel's methods to prove new theorems of "ubiquitous pathology" for classical and quantum lattice systems. The main result is the following: Let $\Phi$ be any interaction and $\varrho$ be any translation-invariant equilibrium state for $\Phi$ (extremal or not). Then there exists a sequence $\left\{\Phi_{k}\right\}$ of interactions converging to $\Phi$, having extremal (or even unique) translationinvariant equilibrium states $\varrho_{k}$, such that $\left\{\varrho_{k}\right\}$ converges to $\varrho$. In certain situations the perturbations $\Phi_{k}-\Phi$ can be chosen to lie in a cone of "antiferromagnetic pair interactions." I discuss the connection with results of Daniëls and van Enter, and point out an application to the one-dimensional ferromagnetic Ising model with $1 / r^{2}$ interaction (Thouless effect).
\end{abstract}

\section{Introduction}

Israel $[1,2]$ has recently introduced elegant abstract methods for the study of classical or quatntum lattice systems in statistical mechanics with general translation-invariant interaction. Two of his results are quite surprising, for they assert that situations generally considered to be "pathological" are in fact ubiquitous :

(a) Let $\varrho_{1}, \ldots, \varrho_{n}$ be any finite family of ergodic translation-invariant states with finite mean entropy. Then there exists some interaction $\Phi$ (in a certain Banach space $\mathscr{B}$ of interactions) for which all these states are equilibrium states.

(b) There is a dense set of interactions in $\mathscr{B}$ each of which has uncountably 1 many ergodic equilibrium states $^{2}$ (i.e. uncountably many pure phases)!

1 In fact, the cardinality is exactly that of the continuum. This is because the extreme points of a metrizable compact convex set are a $G_{\delta}$ [2, Lemma IV.3.1], hence a Borel set; and it can be shown, without invoking the continuum hypothesis, that every uncountable Borel (or even analytic) set in a complete separable metric space has cardinality exactly that of the continuum [3]

2 The proof of Lemma V.2.3 in [2] is incomplete: it needs the additional remark that the set $\left\{\varrho: F(\varrho) \equiv P\left(\Phi_{0}\right)+\varrho\left(A_{\Phi_{0}}\right)-s(\varrho)<\delta\right\}$ is dense in the set $\{\varrho: F(\varrho) \leqq \delta\}$. To see this, assume that $F\left(\varrho_{0}\right)=\delta$; then taking some $\varrho_{1}$ such that $F\left(\varrho_{1}\right)=0$ [i.e., $\varrho_{1}$ is an equilibrium state for $\Phi_{0}$ ] and letting $\varrho_{t}=(1-t) \varrho_{0}$ $+t \varrho_{1}$, we have $\varrho_{t} \rightarrow \varrho_{0}$ as $t \rightarrow 0$ and $F\left(\varrho_{t}\right)<\delta$ for $t>0$ by the convexity (actually affineness) of $F$. I thank Professor Israel for supplying this observation in response to my query, and for giving me permission to include it here 
It may be objected, however, that these two results are devoid of physical content because the interaction space $\mathscr{B}$ is too large. For example, in the classical case it is reasonable to consider the Dobrushin-Lanford-Ruelle (DLR) theory of Gibbs states [4-9] as fundamental, and the theory of invariant equilibrium states as tangents to the pressure as derived. Now it turns out $[2,9]$ that the DLR equations can sensibly be defined only for interactions in a space $\mathscr{B}$ strictly smaller than $\mathscr{B}$. Thus, it is reasonable to argue that only interactions in $\tilde{\mathscr{B}}$ are physically relevant ${ }^{3}$. But in $\tilde{\mathscr{B}}$, unlike $\mathscr{B}$, result (a) is demonstrably false [2, Sect. III.4], and result (b) is presumably false (in any case, the proof fails in $\tilde{\mathscr{B}}$ ).

In this paper I shall use Israel's methods to prove yet another theorem of "ubiquitous pathology." But unlike the two results quoted above, this one cannot be explained away as unphysical: it asserts the existence of Ising models with pair interactions (albeit quite long-range ones) having surprising properties. Similar results have been obtained, also using Israel's methods, by Daniëls and van Enter [10-12].

Let $\left\{\Phi_{k}\right\}$ be a sequence of interactions converging in a suitable sense to an interaction $\Phi$, and for each $k$ let $\varrho_{k}$ be an extremal (=ergodic) translation-invariant equilibrium state ("pure phase") for $\Phi_{k}$. Assume that the states $\left\{\varrho_{k}\right\}$ converge in a suitable sense to a state $\varrho$. Clearly $\varrho$ is a translation-invariant equilibrium state for $\Phi$. But must it be a pure phase? One's first (naive) conjecture is that the answer is yes; one might even conjecture that, as a general fact about convex functions, a limit in the above sense of extremal tangent functionals is necessarily extremal. In fact, this is not the case, as the following simple example shows: on $\mathbb{R}^{2}$, let

$$
f\left(x_{1}, x_{2}\right)=\left(x_{1}^{2}+x_{2}^{4}\right)^{1 / 2} \text {. }
$$

Then at each point $\left(x_{1}, x_{2}\right)=(0, \alpha)$ with $\alpha \neq 0$, the function $f$ has the unique tangent functional $(\nabla f)(0, \alpha)=(0,2 \alpha)$; these converge to $(0,0)$ as $\alpha \rightarrow 0$. On the other hand, at the point $\left(x_{1}, x_{2}\right)=(0,0), f$ has the two extremal tangent functionals $( \pm 1,0)$; thus $(0,0)$ is a non-extremal tangent functional there.

Still, one might think that this kind of behavior, while mathematically possible, does not occur in statistical mechanics. Indeed, a system having a free energy (or pressure) of the form (1.1) would violate the Gibbs phase rule as usually formulated $[2,13,14]$. Of course, the Gibbs phase rule is only a heuristic guide; it is not, at present, a rigorous theorem [10]. Still, one might surmise that it is "usually" valid - that violations of the phase rule are rare "pathologies."

In this paper I prove the exact opposite - that violations of the phase rule are not rare but are in fact ubiquitous. To be precise, I prove the following (Theorem 2.1 and Corollary 2.2): Let $\Phi$ be any interaction and $\varrho$ be any translation-invariant equilibrium state for $\Phi$, extremal or not. Then there exists a sequence $\left\{\Phi_{k}\right\}$ of interactions converging to $\Phi$, having extremal (or even unique) translationinvariant equilibrium states $\varrho_{k}$, such that $\left\{\varrho_{k}\right\}$ converges to $\varrho$. Actually, this result holds in the large Banach space $\mathscr{B}$, so it is open to the objections noted previously. But a related result (Theorem 2.3) shows that in many cases the interactions $\Phi_{k}$ can be taken to lie in the smaller space $\tilde{\mathscr{B}}$; indeed, in the common case in which $\Phi$ has

3 Note also that the pressure can be defined in $\mathscr{B}$ only for free boundary conditions; if one wishes to allow nontrivial boundary conditions, one must restrict attention to interactions in $\tilde{\mathscr{B}}$ [2, pp. 13-14] 
exactly two extremal translation-invariant equilibrium states, having different values of the spontaneous magnetization, the perturbations $\Phi_{k}-\Phi$ can be chosen to be antiferromagnetic pair interactions (albeit of quite long range).

I should emphasize that quite similar behavior has been found previously by Daniëls and van Enter [10-12]. Moreover, their results, while less general than those of the present paper, have a clear physical interpretation (a virtue which is unfortunately not shared by the abstract proofs given here). The construction in [11] is particularly transparent ${ }^{4}$ : Let $\Phi_{0}$ be the interaction for the 2-dimensional Ising model at some temperature $T$ below the critical temperature, and let $\Psi$ be a certain (explicitly given) long-range antiferromagnetic pair interaction. Then van Enter [11] shows that for $\lambda>0$, the interaction $\Phi_{0}+\lambda \Psi$ never has a mixing translation-invariant equilibrium state with nonzero magnetization. The proof is a simple energy argument (see [15] for related ideas): given any translationinvariant state $\varrho$ having $\lim _{|x| \rightarrow \infty} \varrho\left(\sigma_{0} \sigma_{x}\right)=M^{2}>0$, one can construct a new state $\tilde{\varrho}_{N}$ by flipping the spins in bands of width $N$ (and then averaging over translations). Now $\tilde{\varrho}_{N}$ has the same entropy as $\varrho$, but it has lower energy with respect to $\Phi_{0}+\lambda \Psi$ for any $\lambda>0$ (provided that $N=N(\lambda)$ is chosen sufficiently large), so $\varrho$ cannot have been an equilibrium state for $\Phi_{0}+\lambda \Psi$. Presumably the physical picture is that the equilibrium state(s) for $\Phi_{0}+\lambda \Psi$ has large "domains" inside which the state looks roughly like the + or - state for $\Phi_{0}$, and as $\lambda \rightarrow 0$ the domains get larger, so that the limit is a half-and-half mixture of the + and - states. Indeed, if van Enter's theorem could be strengthened to remove the qualifier "mixing" (or equivalently, to replace it by "ergodic"), this conjecture would follow from the fact that the only translation-invariant equilibrium state of the 2-dimensional Ising model which has zero magnetization is the half-sum of the + and - states [16]. ${ }^{5}$. It would be of interest, therefore, to strengthen van Enter's theorem in this way, thereby giving an explicit example of the general phenomenon established in the present paper.

Thus, what I term in the title of this paper a "surprise" perhaps ought not to be surprising at all, once one has absorbed the message of [10-12]. In any case, the results of [10-12] together with those of the present paper indicate that the phase diagrams of lattice systems with general (long-range) interaction must be far more complicated than the naive Gibbs phase rule would suggest.

Finally, it is worth noting that at least one other well-known model - the onedimensional ferromagnetic Ising model with coupling $J / r^{2}-$ exhibits the type of behavior here at issue. In this model, it is believed that:

(i) There is spontaneous magnetization at sufficiently low temperature (large $J)$;

(ii) The spontaneous magnetization $M(J)$ exhibits a discontinuous jump at the critical coupling $J_{c}$, i.e.

$$
M(J)=\left\{\begin{array}{lll}
0 & \text { for } & J<J_{c} \\
M(J) & \text { for } & J \geqq J_{c}
\end{array}\right.
$$

with $M\left(J_{c}\right)>0$ (the "Thouless effect"); and

$4 \quad$ I am indebted to Professor Israel for this lucid explanation of [11]

5 This is true also for the $d$-dimensional Ising model, for all but at most a countable set of temperatures [17] and for all sufficiently low temperatures [18] 
(iii) The free energy density (= pressure) $F(J)$ is infinitely differentiable in $J$ as $J$ passes through $J_{c}$.

These beliefs result from a renormalization-group "solution" of the model [19-21]; in addition, (i) has now been rigorously proven [22], and (ii) is supported by quite general entropy-energy arguments [23, 15] (but see [24]). Now (ii) implies that, at $J_{c}$, the + and - boundary-condition states are distinct pure phases; while (iii) implies, by a theorem of Lebowitz [17], that at $J_{c}$ there are at most two pure phases. Thus, the state obtained by letting $J \uparrow J_{c}$ must be $\frac{1}{2}\left(\langle\cdot\rangle_{+, J_{c}}+\langle\cdot\rangle_{-, J_{c}}\right)$; in other words, a limit of pure (in fact unique) phases in the sense described above is a non-pure phase.

\section{Ubiquitous Failure of the Gibbs Phase Rule}

We assume that the reader is familiar with the results of Israel [2], whose notation we follow; for a brief summary, see [10].

Theorem 2.1. Let $\Phi_{0} \in \mathscr{B}$, and let $\varrho_{0}$ be an invariant equilibrium state for $\Phi_{0}$ (not necessarily extremal). Now let $D_{0}$ be a subset of $\mathscr{B}$ which is dense in a neighborhood of $\Phi_{0}$, and for each $\Phi \in D_{0}$ let $T(\Phi)$ be some nonempty set of invariant equilibrium states for $\Phi$. Then there exists a sequence of interactions $\Phi_{k} \in D_{0}$ and states $\varrho_{k} \in T\left(\Phi_{k}\right)$ such that $\Phi_{k} \rightarrow \Phi_{0}$ (in norm) and $\varrho_{k} \rightarrow \varrho_{0}$ (in weak-* sense).

Proof. By [2, Lemma IV.3.2], there exists a sequence $\left\{\hat{\varrho}_{k}\right\}$ of ergodic translationinvariant states such that $\hat{\varrho}_{k} \rightarrow \varrho_{0}$ in weak-* sense and $s\left(\hat{\varrho}_{k}\right) \downarrow s\left(\varrho_{0}\right)$. Since $\varrho_{0}$ is an invariant equilibrium state for $\Phi_{0}$, we have

$$
P\left(\Phi_{0}\right)+\varrho_{0}\left(A_{\Phi_{0}}\right)-s\left(\varrho_{0}\right)=0
$$

(Gibbs variational equality). Thus

$$
P\left(\Phi_{0}\right)+\hat{\varrho}_{k}\left(A_{\Phi_{0}}\right)-s\left(\hat{\varrho}_{k}\right) \rightarrow 0
$$

as $k \rightarrow \infty$; by passing to a subsequence we can assume that

$$
P\left(\Phi_{0}\right)+\hat{\varrho}_{k}\left(A_{\Phi_{0}}\right)-s\left(\hat{\varrho}_{k}\right) \leqq 1 / k^{2} \text {. }
$$

Then by the Bishop-Phelps theorem [25] in Israel's form [26, 1, 2], there exists, for each $k$, an interaction $\tilde{\Phi}_{k} \in \mathscr{B}$ and an invariant equilibrium state $\tilde{\varrho}_{k}$ for $\tilde{\Phi}_{k}$ such that

$$
\left\|\tilde{\Phi}_{k}-\Phi_{0}\right\| \mid \leqq 1 / k
$$

and

$$
\left\|\tilde{\varrho}_{k}-\hat{\varrho}_{k}\right\| \leqq 1 / k \text {. }
$$

But by the argument in [2, Theorem V.2.2] it follows that $\hat{\varrho}_{k}$ is also an invariant equilibrium state for $\tilde{\Phi}_{k}$ (provided that $k>1$ ); and since $\hat{\varrho}_{k}$ is ergodic, it is an extremal point of the set of invariant equilibrium states for $\tilde{\Phi}_{k}$.

To complete the proof we utilize a generalization of a theorem of Lanford and Robinson [27]; this result is stated and proven in the Appendix. We first equip the set of invariant states with a metric $d$ inducing the weak-* topology; this is possible because $C(\Omega)$ [classical case] or $\mathscr{U}$ [quantum case] is separable (as is $\mathscr{B}$ ). 
Then conclusion (c) of Theorem A.1 implies that (for sufficiently large $k$ ) we can choose $\Phi_{k} \in D_{0}$ and $\varrho_{k} \in T\left(\Phi_{k}\right)$ such that

$$
\left\|\Phi_{k}-\tilde{\Phi}_{k}\right\| \leqq 1 / k
$$

and

$$
d\left(\varrho_{k}, \hat{\varrho}_{k}\right) \leqq 1 / k .
$$

It follows from (2.2)-(2.4) and the definition of $\hat{\varrho}_{k}$ that $\Phi_{k} \rightarrow \Phi_{0}$ in norm and $\varrho_{k} \rightarrow \varrho_{0}$ in weak-* sense.

Corollary 2.2. Let $\Phi_{0} \in \mathscr{B}$, and let $\varrho_{0}$ be an invariant equilibrium state for $\Phi_{0}$ (not necessarily extremal). Then there exists a sequence of interactions $\Phi_{k} \in \mathscr{B}$ having unique invariant equilibrium states $\varrho_{k}$ such that $\Phi_{k} \rightarrow \Phi_{0}$ (in norm) and $\varrho_{k} \rightarrow \varrho_{0}$ (in weak-* sense).

Proof. Since $\mathscr{B}$ is separable, Mazur's theorem [28] (see also [29, 30]) implies that the set of interactions having a unique equilibrium state is dense in $\mathscr{B}$ (in fact, a dense $G_{\delta}$ ). Hence Theorem 2.1 applies.

The meaning of Corollary 2.2 is best understood by comparing it with the (superficially similar) theorem of Lanford and Robinson [27]. The LanfordRobinson theorem, which is a general result about convex functions, states that the set of tangent functionals at any given point is the closed convex hull of the set of limits of unique tangent functionals at nearby points. Corollary 2.2, by contrast, is a special fact about a particular class of convex functions (the pressure in certain statistical-mechanical systems): it asserts that for these apparently rather strange convex functions the words "closed convex hull" in the Lanford-Robinson theorem may be omitted.

Of course, since Theorem 2.1 and Corollary 2.2 refer to the unphysically large Banach space $\mathscr{B}$ of interactions, they are subject to the objections noted in the Introduction. But by using Israel's generalization of the Bishop-Phelps theorem [2, Corollaries V.1.2 and V.3.1], we can arrange for the perturbations $\Phi_{k}-\Phi_{0}$ to lie in a cone of "antiferromagnetic pair interactions" (with possible "magnetic field"); in particular, if $\Phi_{0}$ lies in the physically reasonable space $\tilde{\mathscr{B}}$, then so does $\Phi_{k}$. We can no longer demand (as in Corollary 2.2) that the $\varrho_{k}$ be unique invariant equilibrium states for $\Phi_{k}$, but they are in any case extremal.

The proof of the following theorem is essentially identical to that of [2, Theorem V.3.2] with a few signs changed. It is valid for both classical and quantum systems; we use the notation of the quantum system.

Definition. A family $S \subset \mathscr{U}$ of observables is said to separate the invariant equilibrium states at $\Phi_{0}$ if, given any two such states $\varrho_{1}$ and $\varrho_{2}$, the equality $\varrho_{1}(A)$ $=\varrho_{2}(A)$ for all $A \in S$ implies that $\varrho_{1}=\varrho_{2}$.

Theorem 2.3. Let $\Phi_{0} \in \mathscr{B}$, and let $\varrho_{0}$ be an invariant equilibrium state for $\Phi_{0}$ (not necessarily extremal). Let $S$ be a family of self-adjoint finite-range observables, and for each $A \in S$ let $\Lambda_{A}$ be a finite subset of the lattice such that $A \in \mathscr{U}_{\Lambda_{A}}$. Now let $\mathscr{F}$ be 
the closed convex cone in $\mathscr{B}$ generated by interactions $\Psi \in \mathscr{B}$ of the form

$$
\begin{aligned}
& \Psi\left(i+\Lambda_{A}\right)=h \tau_{i} A, \quad(\text { r real }), \\
& \Psi\left(\left(i+\Lambda_{A}\right) \cup\left(j+\Lambda_{A}\right)\right)=J(i-j)\left(\tau_{i} A\right)\left(\tau_{j} A\right) \quad(J \geqq 0), \\
& \Psi(Y)=0 \quad \text { for all other } Y,
\end{aligned}
$$

with $A \in S$. In the quantum case, to make sure that $\left(\tau_{i} A\right)\left(\tau_{j} A\right)$ is self-adjoint, we require in addition that $J(i)=0$ unless $\left(i+\Lambda_{A}\right) \cap \Lambda_{A}=\emptyset$.

Then there exists a sequence of interactions $\Phi_{k} \in \Phi_{0}+\mathscr{F}$ having extremal (=ergodic) invariant equilibrium states $\varrho_{k}$ such that $\Phi_{k} \rightarrow \Phi_{0}$ (in norm) and $\varrho_{k} \rightarrow \varrho$ (in weak-* sense), where $\varrho$ is an invariant equilibrium state for $\Phi_{0}$ satisfying $\varrho(A)$ $=\varrho_{0}(A)$ for all $A \in S$. In particular, if $S$ separates the invariant equilibrium states at $\Phi_{0}$, then $\varrho=\varrho_{0}$.

Proof. As in the proof of Theorem 2.1, there exists a sequence $\left\{\hat{\varrho}_{k}\right\}$ of ergodic translation-invariant states such that $\hat{\varrho}_{k} \rightarrow \varrho_{0}$ in weak-* sense and (2.1) is satisfied. Then by Israel's generalization of the Bishop-Phelps theorem [2, Corollary V.3.1], there exists, for each $k$, an interaction $\Phi_{k} \in \Phi_{0}+\mathscr{F}$ and an invariant equilibrium state $\tilde{\varrho}_{k}$ for $\Phi_{k}$ such that

$$
\begin{gathered}
\left\|\Phi_{k}-\Phi_{0} \mid\right\| \leqq k^{-1}, \\
\left|\varrho_{k}(A)-\varrho_{k}(A)\right| \leqq k^{-1}\|A\|, \\
\tilde{\varrho}_{k}\left(A \tau_{i} A\right) \leqq \varrho_{k}\left(A \tau_{i} A\right)+k^{-1}\|A\|^{2},
\end{gathered}
$$

for all $A \in S$ and all $i$ satisfying $\left(i+\Lambda_{A}\right) \cap \Lambda_{A}=\emptyset$. In particular, if we let

$$
c_{n}(A)=\frac{1}{\left|C_{n}\right|} \sum_{i \in C_{n}} \tau_{i} A
$$

where $C_{n}$ is a cube of side $n$, then (2.7) implies that

$$
\lim _{n \rightarrow \infty} \tilde{\varrho}_{k}\left(c_{n}(A)^{2}\right) \leqq \lim _{n \rightarrow \infty} \hat{\varrho}_{k}\left(c_{n}(A)^{2}\right)+k^{-1}\|A\|^{2}
$$

for all $A \in S$. (Note that the limits exist, by the ergodic theorem.)

Now since $\tilde{\varrho}_{k}$ is an invariant equilibrium state for $\Phi_{k}$, it has an ergodic decomposition

$$
\tilde{\varrho}_{k}=\int \varrho^{\prime} d \tilde{\mu}_{k}\left(\varrho^{\prime}\right),
$$

where $\tilde{\mu}_{k}$ is a probability measure supported on the ergodic invariant equilibrium states for $\Phi_{k}$. Since the $\varrho^{\prime}$ are ergodic, we have

$$
\lim _{n \rightarrow \infty} \varrho^{\prime}\left(c_{n}(A)^{2}\right)=\varrho^{\prime}(A)^{2}
$$

for each $A \in \mathscr{U}$. Thus

$$
\begin{aligned}
\lim _{n \rightarrow \infty} \tilde{\varrho}_{k}\left(c_{n}(A)^{2}\right) & =\lim _{n \rightarrow \infty} \int \varrho^{\prime}\left(c_{n}(A)^{2}\right) d \tilde{\mu}_{k}\left(\varrho^{\prime}\right) \\
& =\int \varrho^{\prime}(A)^{2} d \tilde{\mu}_{k}\left(\varrho^{\prime}\right)
\end{aligned}
$$


by (2.10) and the dominated convergence theorem. On the other hand,

$$
\begin{aligned}
\lim _{n \rightarrow \infty} \hat{\varrho}_{k}\left(c_{n}(A)^{2}\right) & =\hat{\varrho}_{k}(A)^{2} \\
& \leqq 2 \hat{\varrho}_{k}(A) \tilde{\varrho}_{k}(A)-\hat{\varrho}_{k}(A)^{2}+4 k^{-1}\|A\|^{2} \\
& =\int\left[2 \hat{\varrho}_{k}(A) \varrho^{\prime}(A)-\hat{\varrho}_{k}(A)^{2}\right] d \tilde{\mu}_{k}\left(\varrho^{\prime}\right)+4 k^{-1}\|A\|^{2}
\end{aligned}
$$

for all $A \in S$, by ergodicity of $\hat{\varrho}_{k},(2.6)$ and (2.9). Combining (2.8),(2.11), and (2.12), we get

$$
\int\left[\varrho^{\prime}(A)-\hat{\varrho}_{k}(A)\right]^{2} d \tilde{\mu}_{k}\left(\varrho^{\prime}\right) \leqq 5 k^{-1}\|A\|^{2}
$$

for all $A \in S$.

Now let $\left\{\tilde{A}_{j}\right\}$ be a countable (or finite) dense subset of $S$ (which exists because $S \subset \mathscr{U}$ is a separable metric space), and define

$$
A_{j}=2^{-j}\left(\left\|\tilde{A}_{j}\right\|+1\right)^{-1} \tilde{A}_{j}
$$

so that $\sum_{j}\left\|A_{j}\right\|^{2} \leqq 1$. Applying (2.13) to the $\left\{A_{j}\right\}$ and summing over $j$ (using the monotone convergence theorem), we get

$$
\int \sum_{j}\left[\varrho^{\prime}\left(A_{j}\right)-\hat{\varrho}_{k}\left(A_{j}\right)\right]^{2} d \tilde{\mu}_{k}\left(\varrho^{\prime}\right) \leqq 5 k^{-1} .
$$

Hence there must exist at least one ergodic invariant equilibrium state $\varrho_{k}$ for $\Phi_{k}$ such that

$$
\sum_{j}\left[\varrho_{k}\left(A_{j}\right)-\hat{\varrho}_{k}\left(A_{j}\right)\right]^{2} \leqq 5 k^{-1} .
$$

Thus, for each $j$, we have

$$
\lim _{k \rightarrow \infty} \varrho_{k}\left(A_{j}\right)=\lim _{k \rightarrow \infty} \hat{\varrho}_{k}\left(A_{j}\right)=\varrho_{0}\left(A_{j}\right) ;
$$

and by density the same holds for all $A \in S$. By passing if necessary to a subsequence we can also arrange that the $\varrho_{k}$ converge in weak-* sense to an invariant state $\varrho$; and by (2.5), $\varrho$ must be an invariant equilibrium state for $\Phi_{0}$.

All of the above results are ultimately based on the fact that the set $E^{I}$ of translation-invariant states is particularly badly-behaved: its extreme points form a dense subset. Amusingly, it turns out $[31,32]$ that $E^{I}$ is the unique (up to affine homeomorphism) metrizable Choquet simplex with this proerty (except for the one-point set)!

Finally, it is worth remarking that the Bishop-Phelps theorem - which is the main tool in all of the results of the present paper - has been refined and generalized in numerous directions [33-36]. It would be interesting to know whether any of these generalizations can be applied usefully to statistical mechanics.

\section{Appendix. A Theorem on Convex Functions}

Let $X$ be a real Banach space, and $U$ an open subset of $X$. Let $f$ be a real-valued convex function on $U$. A linear functional $l \in X^{*}$ is said to be a tangent functional 
(or subgradient) to $f$ at the point $x_{0} \in U$ if

$$
f(x) \geqq f\left(x_{0}\right)+l\left(x-x_{0}\right)
$$

for all $x \in U$. The set of all tangent functionals to $f$ at $x_{0}$ is denoted by $\partial f\left(x_{0}\right)$. It follows immediately from (A.1) that $\partial f\left(x_{0}\right)$ is a convex, weak-* closed subset of $X^{*}$. Moreover, if $f$ is continuous, then $\partial f\left(x_{0}\right)$ is non-empty (by the Hahn-Banach theorem) and weak-* compact (by the local Lipschitz property of $f[2$, Lemma VI.2.1], which implies that $\partial f\left(x_{0}\right)$ is bounded). Indeed, this local Lipschitz property implies that $\partial f[U] \equiv \bigcup_{x \in U} \partial f(x)$ is bounded for some neighborhood $U$ of $x_{0}$.

We now prove a slight generalization of a theorem of Lanford and Robinson [27]. If $S$ is a convex subset of $X^{*}$, we denote by ext $S$ the set of all extreme points of $S$.

Theorem A.1. Let $U$ be an open subset of a real Banach space $X$, and let $f: U \rightarrow \mathbb{R}$ be continuous and convex. Let $x_{0} \in U$, let $D_{0}$ be a subset of $U$ which dense in $a$ neighborhood of $x_{0}$, and for each $x \in D_{0}$ let $T(x)$ be a nonempty subset of $\partial f(x)$.

Define

$Z=Z\left(f, x_{0}, D_{0}\right)=\left\{l \in X^{*}:\right.$ there exist nets $x_{\alpha} \rightarrow x_{0}$

(in norm) and $l_{\alpha} \rightarrow l$ (in weak-* topology)

such that $x_{\alpha} \in D_{0}$ and $l_{\alpha} \in T\left(x_{\alpha}\right)$ for all $\left.\alpha\right\}$

and let $Z_{\text {seq }}$ be defined analogously using sequences instead of nets. Then:

(a) $Z$ is a weak-* compact subset of $X^{*}$.

(b) $\overline{c o}(Z)$, the weak-* closed convex hull of $Z$, is weak-* compact and equals $\partial f\left(x_{0}\right)$.

(c) $Z$ contains $\overline{\operatorname{ext} \partial f\left(x_{0}\right)}$.

(d) If $X$ is separable, then $Z=Z_{\text {seq }}$.

In particular, if $X$ is a Banach space which has an equivalent norm such that the dual norm $\|\cdot\|_{X^{*}}$ is strictly convex (for example, if $X$ is separable or reflexive), then we can take $D_{0}=D_{1}(f) \backslash\left\{x_{0}\right\}$, where

$$
D_{1}(f)=\{x \in U: \partial f(x) \text { has exactly one element }\} .
$$

Proof. Let $U_{0}$ be a neighborhood of $x_{0}$ such that $\partial f\left[U_{0}\right]$ is bounded; then so is $Z \subset \overline{\partial f\left[U_{0}\right]}$. Moreover, $Z$ is easily seen to be weak-* closed. By the BanachAlaoglu theorem, this proves (a). Likewise, $\overline{c o}(Z)$ is bounded and weak-* closed, hence weak-* compact. Also, it follows easily from (A.1) that $Z \subset \partial f\left(x_{0}\right)$ and hence also $\overline{c o}(Z) \subset \partial f\left(x_{0}\right)$.

Now assume that (b) is false, i.e. that there exists $l_{0} \in \partial f\left(x_{0}\right)$ with $l_{0} \notin \overline{c o}(Z)$. Then by the Hahn-Banach theorem there exists $y_{0} \in X$ such that $l_{0}\left(y_{0}\right)>l\left(y_{0}\right)$ for all $l \in \overline{c o}(Z)$. Now for $n$ sufficiently large, $x_{0}+\frac{1}{n} y_{0} \in U_{0} \cap \overline{D_{0}}$, so we can choose $x_{n} \in U_{0} \cap D_{0}$ such that $z_{n} \equiv x_{n}-\left(x_{0}+\frac{1}{n} y_{0}\right)$ has norm at most $1 / n^{2}$. Now choose any 
$l_{n} \in T\left(x_{n}\right)$. Then $\left\langle l_{n}-l_{0}, x_{n}-x_{0}\right\rangle \geqq 0$ by a simple computation using (A.1), hence

$$
l_{n}\left(y_{0}\right)-l_{0}\left(y_{0}\right) \geqq-n\left\langle l_{n}-l_{0}, z_{n}\right\rangle \geqq-\frac{K}{n},
$$

where $K=\operatorname{diam} \partial f\left[U_{0}\right]<\infty$. Since $\left\{l_{n}\right\}$ is bounded, we can extract a weak-* convergent subnet $\left\{l_{n(\alpha)}\right\} \rightarrow l \in Z$ and conclude that $l\left(y_{0}\right) \geqq l_{0}\left(y_{0}\right)$, a contradiction. This proves (b).

(c) follows from (a)-(b) and Milman's converse to the Krein-Milman theorem. (d) follows from the boundedness of $\partial f\left[U_{0}\right]$ and the metrizability of the weak-* topology on bounded subsets of $X^{*}$ when $X$ is separable. The final assertion follows from a result of Asplund [29], which states that $D_{1}$ contains a dense $G_{\delta}$ subset of $U$. (This was proven earlier by Mazur [28] for the case of $X$ separable.)

Remarks. 1. This result is contained (without proof) in a note of Shih [37]. Lanford and Robinson [27] proved part (b) in the case $D_{0}=D_{1}(f) \backslash\left\{x_{0}\right\}, X$ separable. My proof of part (b) follows a simplification of their proof, due to Ruelle [9, p. 44], which allows "dense $G_{\delta}$ " to be replaced by "dense." The key additional observation, which is needed in Sect. 2 of the present paper, is that $Z$ contains ext $\partial f\left(x_{0}\right)$; this was already noticed by Preston [8, p. 153]. A complete proof is given here for the reader's convenience.

2. Theorem A.1 can be extended to Fréchet spaces. Also, it holds in the more general framework of monotone mappings $T$ from $X$ to subsets of $X^{*}$; see $[38,39]$.

Acknowledgement. This work had its origin in a joint study, with Barry Simon, of the Thouless effect [15]; in particular, the initial example of type (1.1) is due to him. I thank Professor Simon for many valuable conversations. I also thank Professor Robert Israel for enlightening conversations and correspondence.

\section{References}

1. Israel, R.B.: Existence of phase transitions for long-range interactions. Commun. Math. Phys. 43, 59-68 (1975)

2. Israel, R.B.: Convexity in the theory of lattice gases. Princeton, N.J.: Princeton University Press 1979

3. Kuratowski, K.: Topology, Vol. I. New York, London, Warsaw: Academic Press/PWN-Polish Scientific Publishers, 1966, pp. 447, 479

4. Dobrushin, R.L.: The description of a random field by means of conditional probabilities and conditions of its regularity. Teor. Veroya. Prim. 13, 201-229 (1968) [Theor. Prob. Appl. 13, 197-224 (1968)]

5. Dobrushin, R.L.: Gibbsian random fields for lattice systems with pairwise interactions. Funkt. Anal. Prilozhen. 2, No. 4, 31-43 (1968) [Funct. Anal. Appl. 2, 292-301 (1968)]

6. Dobrushin, R.L.: Prescribing a system of random variables by conditional distributions. Teor. Veroya. Prim. 15, 469-497 (1970) [Theor. Prob. Appl. 15, 458-486 (1970)]

7. Lanford, O.E. III, Ruelle, D.: Observables at infinity and states with short range correlations in statistical mechanics. Commun. Math. Phys. 13, 194-215 (1969)

8. Preston, C.: Random fields. In: Lecture Notes in Mathematics, Vol. 534. Berlin, Heidelberg, New York: Springer 1976

9. Ruelle, D.: Thermodynamic formalism. Reading, MA : Addison-Wesley 1978

10. Daniëls, H.A.M., van Enter, A.C.D.: Differentiability properties of the pressure in lattice systems. Commun. Math. Phys. 71, 65-76 (1980)

11. van Enter, A.C.D.: A note on the stability of phase diagrams in lattice systems. Commun. Math. Phys. 79, 25-32 (1981) 
12. van Enter, A.C.D.: Stability properties of phase diagrams in lattice systems. Proefschrift, Rijksuniversiteit te Groningen (1981)

13. Wightman, A.S.: Convexity and the notion of equilibrium state in thermodynamics and statistical mechanics. Introduction to [2]

14. Ruelle, D.: A heuristic theory of phase transitions. Commun. Math. Phys. 53, 195-208 (1977)

15. Simon, B., Sokal, A.D.: Rigorous entropy-energy arguments. J. Stat. Phys. 25, 679-694 (1981)

16. Messager, A., Miracle-Sole, S. : Equilibrium states of the two-dimensional Ising model in the twophase region. Commun. Math. Phys. 40, 187-196 (1975)

17. Lebowitz, J.L.: Coexistence of phases in Ising ferromagnets. J. Stat. Phys. 16, 463-476 (1977)

18. Gallavotti, G., Miracle-Sole, S.: Equilibrium states of the Ising model in the two-phase region. Phys. Rev. B 5, 2555-2559 (1972)

19. Anderson, P.W., Yuval, G., Hamann, D.R.: Exact results in the Kondo problem. II. Scaling theory, qualitatively correct solution, and some new results on one-dimensional classical statistical models. Phys. Rev. B1, 4464-4473 (1970)

20. Anderson, P.W., Yuval, G.: Some numerical results on the Kondo problem and the inverse-square one-dimensional Ising model. J. Phys. C4, 607-620 (1971)

21. Cardy, J.L.: One-dimensional models with $1 / r^{2}$ interactions. J. Phys. A 14, 1407-1415 (1981)

22. Fröhlich, J., Spencer, T.: The phase transition in the one dimensional Ising model with $1 / r^{2}$ interaction energy. Commun. Math. Phys. 84, 87-101 (1982)

23. Thouless, D.J.: Long-range order in one-dimensional Ising systems. Phys. Rev. 187, 732-733 (1969)

24. Bhattacharjee, J., Chakravarty, S., Richardson, J.L., Scalapino, D.J.: Some properties of a onedimensional Ising chain with an inverse-square interaction. Phys. Rev. B 24, 3862-3865 (1981)

25. Bishop, E., Phelps, R.R.: The support functionals of a convex set. In: Convexity. Proceedings of symposia in pure mathematics, Vol. 7. Providence RI : American Mathematical Society, 1963, pp. $27-35$

26. Brøndsted, A., Rockafellar, R.T.: On the subdifferentiability of convex functions. Proc. Am. Math. Soc. 16, 605-611 (1965)

27. Lanford, O.E. III, Robinson, D.W.: Statistical mechanics of quantum spin systems. III. Commun. Math. Phys. 9, 327-338 (1968)

28. Mazur, S.: Über konvexe Mengen in linearen normierten Räumen. Studia Math. 4, 70-84 (1933)

29. Asplund, E.: Fréchet differentiability of convex functions. Acta Math. 121, 31-47 (1968)

30. Larman, D.G., Phelps, R.R.: Gateaux differentiability of convex functions on Banach spaces. J. London Math. Soc. 20, 115-127 (1979)

31. Lindenstrauss, J., Olsen, G., Sternfeld, Y.: The Poulsen simplex. Ann. Inst. Fourier (Grenoble) 28, no. 1, 91-114 (1978)

32. Olsen, G.H.: On simplices and the Poulsen simplex. In: Functional Analysis: Surveys and recent results II. Proceedings of the conference on functional analysis, Paderborn, Germany, 1979. NorthHolland mathematics studies \# 38 (eds. K.-D. Bierstedt and B. Fuchssteiner). Amsterdam, New York, Oxford: North-Holland 1980, pp. 31-52

33. Phelps, R.R.: Support cones in Banach spaces and their applications. Adv. Math. 13, 1-19 (1974)

34. Ekeland, I.: On the variational principle. J. Math. Anal. Appl. 47, 324-353 (1974)

35. Ekeland, I.: Nonconvex minimization problems. Bull. Am. Math. Soc. 1, 443-474 (1979)

36. Sullivan, F.: A characterization of complete metric spaces. Proc. Am. Math. Soc. 83, 345-346 (1981)

37. Shih Shu-Chung: Remarques sur le gradient généralisé. C.R. Acad. Sci. Paris A 291, 443-446 (1980)

38. Kenderov, P., Robert, R.: Nouveaux résultats génériques sur les opérateurs monotones dans les espaces de Banach. C.R. Acad. Sci. Paris A 282, 845-847 (1976)

39. Sokal, A.D.: Unpublished manuscript (1981)

\section{Communicated by E. Lieb}

Received December 10, 1981 